\title{
Multi-Agent Based Distributed Vehicle Deployment Planning for Shipment Yard of Automobile Assembly Workshop
}

\author{
Lingge Zhang \\ School of Mechanical Engineering, Shanghai Jiao Tong University, Shanghai, 200240, China
}

\begin{abstract}
Vehicle deployment for shipment yard of automobile assembly workshop refers to selecting an appropriate parking slot for a vehicle released to shipment yard from assembly shop when it's parked in the shipment yard for temporary storage. Based on analysis of shipment yard's operation way, a mathematical model of the vehicle deployment planning is established. Each released vehicle or parking slot is embedded with computation and memory capabilities, making each of them an agent. Vehicle deployment plan is made through coordination between agents. A coordination mechanism based on iterative algorithm is established to dynamically update the plan. Case analysis verifies effectiveness of model and algorithm.
\end{abstract}

KEYWORD: Vehicle deployment planning; dynamic; multi-agent; distributed

\section{INTRODUCTION}

In the past few decades, the performance of supply chain and manufacturing decision-making system are significantly improved. But these improvements can't well solve the disturbance caused by a series of dynamic and uncertainty. Solving the problem of dynamic demand the decision system to be responsive and flexible, being able to deal with real time information in a timely manner in order to quickly respond to the dynamic environment.

Traditional way of centralized decision-making is based on a single point of control, so it is difficult to make a rapid response to dynamic environment change. Distributed method with features like adaptability, scalability and reliability (Cao Y et al, 2013), can quickly respond to changing environment. An Agent has the characteristics of autonomy, intelligence, adaptation and co-operation (Leitão P, 2009), so a multi-agent system can deal with disturbance well, making it a good method for distributed decision making.

Outbound logistics of automobile industry is playing a more and more important role in manufacturing market competition. But now, study of outbound logistics is mainly concerned on location and layout of logistics facility (Klose A et al, 2005), finished cars' scheduling and routing (Norstad I et al, 2011) and allocation scheme of logistics storage space (YONGBIN H A N, 2008), etc. Few researches are focused on decision making in shipment yard management process. Cordeau J F (Cordeau J F et al, 2011) analyzes management problem of transshipment terminal for finished cars. Kim J (Kim J et al, 2008) analyzes dynamic truck arrival time for finished car transportation.

This paper presents a multi-agent based distributed vehicle deployment planning, which is used to provide decision support for selection of a parking slot when a car is released to shipment yard.

\section{PROBLEM DEFINITION}

\subsection{Operations of the vehicle deployment process.}

As shown in Fig.1, four operations are included. (1) A yard operator drives vehicle $i$ for temporary spot $T$ to allocated parking slot $s_{i}$ in parking area. (2)The yard operator rides (because of the long distance) back to temporary spot $T$. (3) A truck driver walks (because of the short distance) to the parking slot $s_{i}$ from loading location $L_{i}$. (4) The truck driver drives back to loading location $L i$.

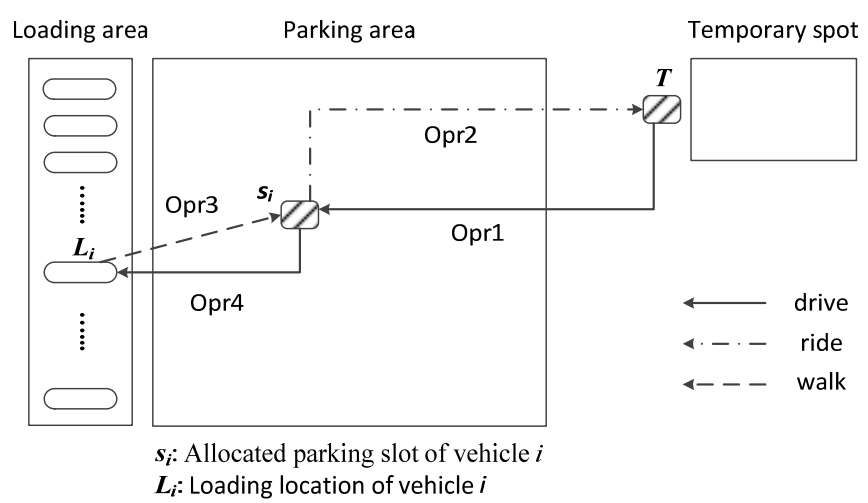

Fig.1 Process for deployment of vehicle I (Kim J et al, 2010) 


\subsection{Measurement of vehicle deployment plan.}

Total operation time of four operations, which is related with distance and speed, is used as the measure. The speed of driving, riding and walking are three constants, and distance varies due to the difference of parking slot allocation.

For two locations $M \quad\left(m_{1}, m_{2}\right)$ and $N\left(n_{1}, n_{2}\right)$, driving distance and riding distance are calculated by

Total_time $\left(s_{i}, L_{i}\right)=\frac{D_{d}\left(T, s_{i}\right)}{v_{d}}+t_{\text {wait }}+\frac{D_{r}\left(s_{i}, T\right)}{v_{r}}+\frac{D_{W}\left(L_{i}, S_{i}\right)}{v_{w}}+\frac{D_{d}\left(s_{i}, L_{i}\right)}{v_{d}}+t_{\text {load }}$

Where $v_{d}, v_{r}$ and $v_{w}$ are speed of driving, riding and walking, $t_{\text {wait }}$ is waiting time of a yard operator for a van, $t_{\text {load }}$ is the loading time a truck driver used to load a car on a truck.
$D_{d}(M, N)=D_{r}(M, N)=\left|m_{1}-n_{1}\right|+\left|m_{2}-n_{2}\right|$

And walk distance is calculated by

$D_{w}(M, N)=\sqrt{\left(m_{1}-n_{1}\right)^{2}+\left(m_{2}-n_{2}\right)^{2}}$

Total operation time of vehicle $i$ with parking slot $s_{i}$ and loading location $L_{i}$ :

\subsection{Current centralized vehicle deployment planning.}

State of parking slots is updated in a manual, periodic way. Allocation of parking slots is based on the initial

$\min \sum_{s \in \text { Set }^{i}}\left\{\left(\frac{D_{d}\left(T, s_{i}\right)}{v_{d}}+t_{\text {wait }}+\frac{D_{r}\left(s_{i}, T\right)}{v_{r}}+\frac{D_{W}\left(L_{i}, s_{i}\right)}{v_{w}}+\frac{D_{d}\left(s_{i}, L_{i}\right)}{v_{d}}+t_{\text {load }}\right)\right\} x_{S}^{i}$

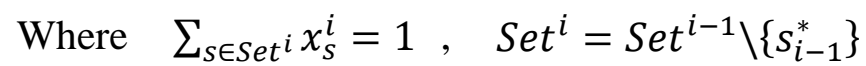
and $x_{s}^{i} \in(0,1) \quad \forall s \in S e t^{i}$. If vehicle $i$ is allocated to parking slot $s$, then the value of $x_{s}^{i}$ is 1 ; or the value of $x_{s}^{i}$ is 0 .

\section{MULTI-AGENT BASED DISTRIBUTED VEHICLE DEPLOYMENT PLANNING}

\subsection{Design of the multi-agent system.}

Two kinds of agents are included.

Parking slot agent: A parking slot agent represents a parking slot, and is responsible for communication with vehicle agents. It has information of its state (idle, allocated or occupied)

Vehicle agent: A vehicle agent represents a released vehicle, and is responsible for obtaining a parking slot. It stores information of loading location, current location and allocated parking slot. state when this period starts. Thus, due to the characteristics of manual operation, even a parking slot becomes idle form occupied, it can't participate in subsequent allocation process in this period. Deployment result is calculated by

$$
\min \sum_{s \in \operatorname{Set}^{i}}\left\{\left(\frac{D_{d}\left(T, s_{i}\right)}{v_{d}}+t_{\text {wait }}+\frac{D_{r}\left(s_{i}, T\right)}{v_{r}}+\frac{D_{W}\left(L_{i}, S_{i}\right)}{v_{w}}+\frac{D_{d}\left(s_{i}, L_{i}\right)}{v_{d}}+t_{\text {load }}\right)\right\} x_{s}^{i}
$$

Where $\operatorname{Set}^{i}$ is the set of idle (neither in occupied nor allocated state) parking slots when vehicle $\mathrm{i}$ is released.

(2) Updating initial deployment planning. At certain time point, if state of a parking slot turns idle from occupied, this parking slot needs to participate in allocation process of the vehicle agent which already having a allocated parking slot but not being moved yet (due to the existence of time waiting for a yard operator).
Vehicle deployment process: (1) When a vehicle is released, initial deployment planning is generated with information of current states of parking slots and the released car. (2) Initial deployment planning is updated based on dynamic, such as change of idle parking slots set. Detailed process is as follows.

(1) Generating initial deployment. When a vehicle is released, this vehicle agent sends calling for tenders' message including loading location. Each idle parking slot participates in the bidding process by calculating its total operation time for this car and then submitting a tender including this operation time to the vehicle agent. The vehicle agent receive these tenders and sends succeeding message to parking slot agent with the shortest operation time and failure message to other parking slot agents participating in the process. State of parking slots is automatically updated in real time through RFID technology. In this way, allocation result of vehicle $i$ can be calculated by

An iterative algorithm is used in this paper to realize the updating process:

A. Parking slot agent $S_{\text {new }}$ submits tenders including total operation time to vehicle agents in $V^{P}$ $\left(V^{P}\right.$ is a set of vehicle agents each of which is already allocated to a parking slot, but not moved from the temporary spot yet).

B. Each vehicle agent in $V^{P}$ calculates the difference value between current total operation time and total operation time provided by $S_{\text {new }}$. If the difference value is positive, the vehicle agent 
sends a succeeding message to vehicle agent $S_{\text {new }}$. Otherwise, it sends a failure message.

C. If vehicle agent $S_{\text {new }}$ doesn't receive any message including difference value, algorithm terminates; otherwise, go to 3 .

D. Vehicle agent $S_{\text {new }}$ sends a confirmation message to vehicle agent providing the biggest difference value, and sends a failure message to other participated vehicle agents.

E. Vehicle agent $i^{*}$ receives the confirmation message, and then sends a deallocating message to current allocated parking slot agent. Set $S_{\text {new }}$ to be the new current allocated parking slot.

F. If the number of iterations reaches limit Max, algorithm terminates; otherwise, go to 6 .

G. Set $S_{\text {new }}=S_{i^{*}}$, go to 1 .

\section{CASE ANALYSIS}

\subsection{Experimental setup.}

Structure of shipment yard in this case is shown if Fig.2. Speed of driving and riding is 9 and 7 times of walking speed, respectively. $T_{\text {wait }}$ and $T_{\text {load }}$ are uniformly distributed date with mean value of 8,10 and distribution interval of $[7,9]$ and $[8,12]$, respectively. Use matlab2010b for experiment programming. Total number of the released car is set to 20000. Each scheme (current scheme, initial scheme and updated scheme) runs 15 times.

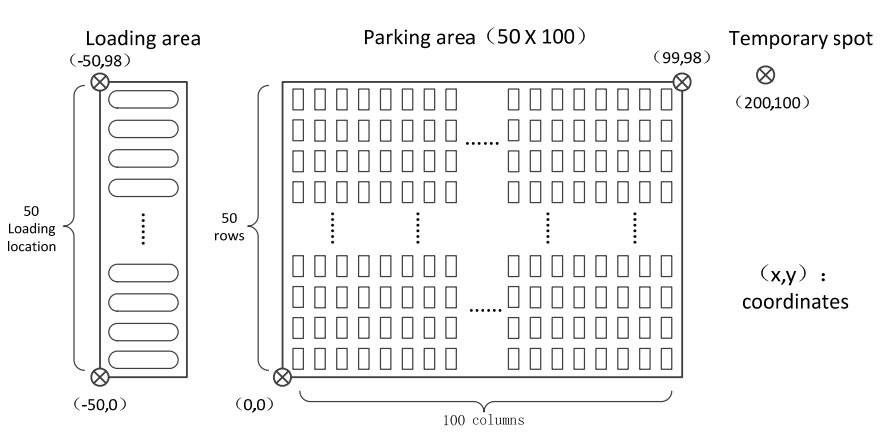

Fig.2 Structure of shipment yard in experiments

\subsection{Experiment result.}

Average total operation time of three schemes in 15 times is shown in table. 1 below. Average parking times of each parking slot column are shown in Fig.3.

Table.1 Total operation time result

\begin{tabular}{|c|c|c|c|c|c|c|c|}
\hline & \multicolumn{5}{|c|}{ Average total operation time } & \multirow[t]{2}{*}{ Mean value } & \multirow[t]{2}{*}{ Reduction ratio compared with last scheme } \\
\hline & 396.35 & 396.75 & 396.48 & 396.73 & 396.71 & & \\
\hline \multirow[t]{3}{*}{ Current scheme } & 396.50 & 396.34 & 396.46 & 396.60 & 396.4 & 396.54 & / \\
\hline & 396.47 & 396.63 & 396.35 & 396.66 & 396.68 & & \\
\hline & 338.49 & 338.25 & 338.38 & 338.48 & 337.82 & & \\
\hline \multirow[t]{3}{*}{ Initial scheme } & 338.47 & 337.95 & 338.26 & 338.05 & 338.20 & 338.24 & $14.7 \%$ \\
\hline & 338.24 & 338.26 & 338.31 & 338.27 & 338.22 & & \\
\hline & 316.37 & 316.42 & 316.18 & 316.25 & 316.30 & & \\
\hline \multirow[t]{2}{*}{ Updated scheme } & 316.82 & 316.21 & 316.31 & 316.38 & 316.49 & 316.33 & $6.5 \%$ \\
\hline & 316.57 & 316.08 & 316.32 & 316.02 & 316.20 & & \\
\hline
\end{tabular}

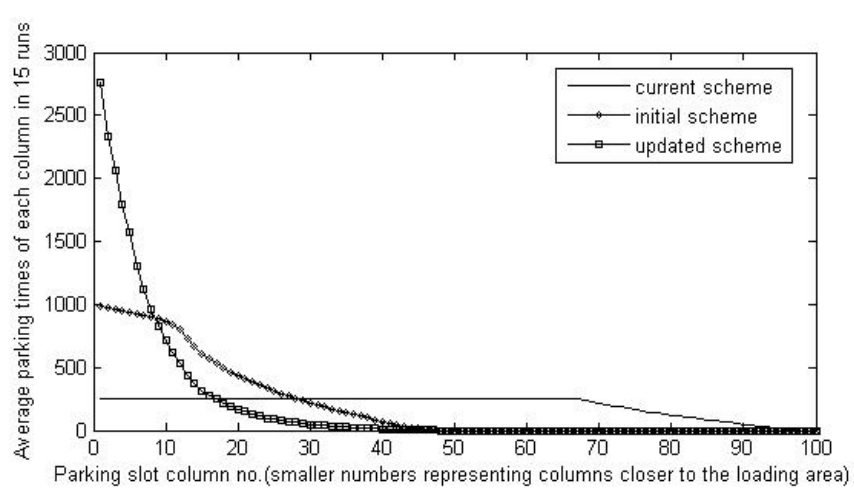

Fig.3 Average parking times of each parking slot column

It can be seen that average total operation time of initial scheme is $14.7 \%$ less than that of current scheme, and average total operation time of updated scheme is $6.5 \%$ less than that of initial scheme. This proves that the model and algorithm in this paper are effective.
From Fig.3, we know that the closer to loading area, the bigger the average parking times of those parking slot columns are. This is due to that the parking slot closer to the loading area can provide a better vehicle deployment performance (less total operation time). In initial scheme and updated scheme, parking times increase significantly in columes near the loading area.

\section{CONCLUSION}

This paper builds the mathematical model and multiagent based distributed decision making system for vehicle deployment planning of automobile assembly workshop's shipment yard, with the goal of minimizing total operation time used for vehicle movement. Case analysis not only verifies effectiveness of model and algorithm, but also provides some direction for shipment yard managers in reducing size of the park- 
ing area. In future research, we can consider more factors in measure of deployment plan and dynamic aspects, and think about better ways to update the initial deployment scheme.

\section{REFERENCES}

Cao Y, Yu W, Ren W, et al. An overview of recent progress in the study of distributed multi-agent coordination[J]. Industrial Informatics, IEEE Transactions on, 2013, 9(1): 427-438.

Cordeau J F, Laporte G, Moccia L, et al. Optimizing yard assignment in an automotive transshipment terminal[J]. European Journal of Operational Research, 2011, 215(1): 149-160.

Kim J, Ok C. Distributed feedback control algorithm for dynamic truck loading scheduling problem [J]. Applied Mathematics and Computation, 2008, 199(1): 275-284.

Kim J, Ok C S, Kumara S, et al. A market-based approach for dynamic vehicle deployment planning using radio frequency identification (RFID) information[J]. International Journal of Production Economics, 2010, 128(1): 235-247.

Klose A, Drexl A. Facility location models for distribution system design [J]. European Journal of Operational Research, 2005, 162(1): 4-29.

Leitão P. Agent-based distributed manufacturing control: A state-of-the-art survey [J]. Engineering Applications of Artificial Intelligence, 2009, 22(7): 979-991.

Norstad I, Fagerholt K, Laporte G. Tramp ship routing and scheduling with speed optimization [J]. Transportation Research Part C: Emerging Technologies, 2011, 19(5): 853-865.

YONGBIN H A N. Efficient yard storage in transshipment container hub ports [D], 2008. 